\title{
BMJ
}

\section{Presentation, pattern, and natural course of severe symptoms, and role of antibiotics and antibiotic resistance among patients presenting with suspected uncomplicated urinary tract infection in primary care: observational study}

\author{
P Little, professor of primary care research, ${ }^{1} \mathrm{R}$ Merriman, medical student, ${ }^{1} \mathrm{~S}$ Turner, study manager, ${ }^{1} \mathrm{~K}$ \\ Rumsby, study data coordinator, ${ }^{1} \mathrm{G}$ Warner, general practitioner, ${ }^{2} \mathrm{~J}$ A Lowes, consultant microbiologist, ${ }^{3} \mathrm{H}$ \\ Smith, professor of primary care, ${ }^{4} \mathrm{C}$ Hawke, public health physician, ${ }^{5} \mathrm{G}$ Leydon, senior postdoctoral research \\ fellow, ${ }^{1} \mathrm{M}$ Mullee, senior lecturer in medical statistics and director of RDSU ${ }^{6} \mathrm{M}$ V Moore, senior lecturer ${ }^{1}$
}

\section{${ }^{1}$ Primary Medical Care Group, Community Clinical Sciences Division (CCS), School of Medicine, University of Southampton: Aldermoor Health Centre, Southampton S016 6ST \\ ${ }^{2}$ Nightingale Surgery, Romsey, S051 7QN \\ ${ }^{3}$ Southampton Universities Hospital Trust Microbiology Laboratory, Southampton General Hospital, Southampton S016 6YD ${ }^{4}$ Brighton and Sussex Medical School, University of Sussex, Brighton BN1 9PX \\ ${ }^{5}$ School of Rural Health, University of Sydney, Orange Campus, PO Box 1191, Orange, NSW, Australia \\ ${ }^{6}$ Community Clinical Sciences Division (CCS), School of Medicine, University of \\ Southampton, Public Health Sciences and Medical Statistics \\ Group, Southampton General Hospital, Southampton S016 6YD Correspondence to: P Little, University of Southampton, Aldermoor Health Centre, Southampton S016 5ST p.little@soton.ac.uk}

Cite this as: $B M J$ 2010;340:b5633 doi:10.1136/bmi.b5633

\section{ABSTRACT}

Objective To assess the natural course and the important predictors of severe symptoms in urinary tract infection and the effect of antibiotics and antibiotic resistance. Design Observational study.

Setting Primary care.

Participants 839 non-pregnant adult women aged 18-70 presenting with suspected urinary tract infection.

Main outcome measure Duration and severity of symptoms. Results 684 women provided some information on symptoms; 511 had both laboratory results and complete symptom diaries. For women with infections sensitive to antibiotics, severe symptoms, rated as a moderately bad problem or worse, lasted 3.32 days on average. After adjustment for other predictors, moderately bad symptoms lasted 56\% longer (incidence rate ratio 1.56 , $95 \%$ confidence interval 1.22 to $1.99, \mathrm{P}<0.001)$ in women with resistant infections; $62 \%$ longer $(1.62,1.13$ to 2.31 , $\mathrm{P}=0.008)$ when no antibiotics prescribed; and $33 \%$ longer $(1.33,1.14$ to $1.56, \mathrm{P}<0.001)$ in women with urethral syndrome. The duration of symptoms was shorter if the doctor was perceived to be positive about diagnosis and prognosis (continuous 7 point scale: $0.91,0.84$ to 0.99 ; $\mathrm{P}=0.021$ ) and longer when the woman had frequent somatic symptoms $(1.03,1.01$ to $1.05, P=0.002$; for each symptom), a history of cystitis, urinary frequency, and more severe symptoms at baseline.

Conclusion Antibiotic resistance and not prescribing antibiotics are associated with a greater than $50 \%$ increase in the duration of more severe symptoms in women with uncomplicated urinary tract infection. Women with a history of cystitis, frequent somatic symptoms (high somatisation), and severe symptoms at baseline can be given realistic advice that they are likely to have severe symptoms lasting longer than three days.

\section{INTRODUCTION}

Urinary tract infections are one of the most common conditions seen in female patients in general practice ${ }^{1}$ and the most common bacterial infection in women. ${ }^{12}$ Urine is the most commonly received specimen in microbiological laboratories, and more than 20\% of isolates are resistant to trimethoprim and cephalosporins and 50\% are resistant to amoxicillin.

\section{Antibiotic resistance}

A recent study in the United Kingdom documented the impact of antibiotic resistance for patients with urinary tract infection caused by Escherichia coli based on retrospective telephone assessment of symptoms, ${ }^{1}$ which limited the ability to accurately assess the pattern and severity of symptoms. A recent prospective study used diaries to document the association of antibiotic resistance with three days' longer duration of milder symptoms and defined symptom resolution as symptoms being labelled a very slight problem or less. ${ }^{4}$ Patients and doctors, however, might be reluctant to alter prescribing decisions based on the duration of mild symptoms so the impact on more meaningful severe symptoms requires clarification. The impact of a resistant infection would be expected to be similar to one in which antibiotics were withheld, assuming microbiological diagnosis is important, which has been questioned recently. ${ }^{5}$ Such a natural "experiment" would provide useful and complementary information to the limited data available for antibiotics from placebo controlled trials. ${ }^{3}$ Finally, no observational study to date has explored whether there are subgroups of symptoms that more clearly respond to antibiotics or assessed and controlled for other factors that might strongly confound the assessment of symptom resolution (such as somatic symptom perception and health anxiety $^{6}$ and variables regarding consultation with the doctor such as a positive approach to the problem). ${ }^{27}$

\section{Natural course}

Limited data from trials suggest that uncomplicated urinary tract infections have a good long term 
prognosis, with a low risk of complications. ${ }^{3}$ A Canadian study characterised the natural course in primary care $^{8}$ but was limited by retrospective telephone interviews and the women were all treated with a 10 day course of ciprofloxacin (a second line treatment in UK practice). ${ }^{9}$ The study did not provide information on the impact of antibiotic resistance or of not providing antibiotics. It is also unclear whether subgroups of symptoms of urinary tract infection are most helped by antibiotics. There is also a paucity of data on the natural course and pattern of symptoms in those presenting with suspected urinary tract infection but with no apparent bacterial growth (that is, so called "urethral syndrome").

We described presentation and the natural course of more severe symptoms in women presenting with suspected urinary tract infection in primary care and the impact of no treatment with antibiotics and of antibiotic resistance. We also documented the key demographic and consultation variables determining the duration and severity of symptoms

\section{METHOD}

This observational study was nested within a diagnostic study ${ }^{10}$ to develop a clinical scoring system for urinary tract infection by using symptoms and dipstick results (but independent of the trial cohort, see associated paper $^{11}$ ). Between January 2002 and February 2005, 117 general practitioners and nurses in 67 practices in the south of England recruited non-pregnant women (aged 18-75) presenting with a suspected uncomplicated urinary tract infection. This group was chosen as they represent the commonest group presenting with suspected urinary tract infection in primary care and also the group in whom antibiotic use is not mandatory.

\section{Exclusions}

We excluded those in whom antibiotic treatment is more definitely indicated (children, men, pregnant women, patients with pyelonephritis), those with nausea, vomiting, or other severe systemic symptoms, and women aged over 75 (in this age group there is a different pattern of symptom reporting $\left.{ }^{1213}\right)$. We also excluded patients with psychotic illnesses or dementia or who needed terminal care as they might be unable to complete the diary.

General practitioners or practice nurses recruited patients with suspected urinary tract infection. The practitioner recorded baseline symptoms, clinical information, and age, sex, and postcode, and noted whether antibiotics were prescribed. They also asked all patients to provide a fresh sample of midstream urine. Each patient kept a daily diary of symptoms, grading severity for up to 14 days: 0 (no symptoms), 1 (very slight problem), 2 (slight problem), 3 (moderately bad problem), 4 (bad problem), 5 (very bad problem), or 6 (as bad as it could be). The symptoms in the diary (dysuria, haematuria, frequency during day and night, "smelly urine," "tummy pain," generally feeling unwell, and restriction of daily activities) were chosen based on the common presenting symptoms of urinary tract infection ${ }^{10}{ }^{14}$ and were collected in a diary that has previously been validated and shown to be sensitive to change for other acute infections. ${ }^{15}$ Patients were also phoned by the research assistant after three days to check there were no problems with completing the questions but were not asked about compliance or to return to the surgery as this could alter their behaviour. A reminder was sent if no diary was returned and, if necessary, a subsequent shorter questionnaire and telephone contact to provide basic information about the duration of symptoms.

When they had completed the diary, patients also completed a validated questionnaire about their perceptions of different aspects of communication in the consultation. ${ }^{27}$ Their perceptions of the doctor's communication were all measured on a scale from 0 (very strongly disagree that the doctor did this) to 6 (very strongly agree)..$^{27}$ They also described enablementthe extent to which they felt enabled to manage both this episode and future episodes ${ }^{16}$ - and completed a somatic symptom inventory (a measure of somatisation $)^{6}$ when the diary was finished. On completion they returned their diaries to the research centre in a freepost envelope.

\section{Laboratory analysis}

The urine samples were transported as in routine practice, and a $10 \mu \mathrm{l}$ sample of urine was cultured on CLED (cysteine lactose electrolyte deficient) agar and incubated overnight at $37^{\circ} \mathrm{C}$. We used EU guidelines, which advocate reporting down to $10^{9}$ cfu (colony forming units) per l or pure growth of $E$ coli but higher counts for more unusual organisms or mixed growths. ${ }^{17}$ When there was no growth or growth not consistent with infection (that is, probable contamination) patients were classified as having "urethral" syndrome. The use of lower colony counts is based on evidence of response to treatment, ${ }^{17}$ further evidence from validation studies, ${ }^{18}$ and consensus internationally ${ }^{17}$; and the latest UK standards also include lower colony counts (www.hpa-standardmethods.org. uk/documents/bsop/ pdf/bsop41.pdf).

\section{Sample size}

Calculation was done for $\alpha=0.05$, and $\beta=0.2$ with the NQuery sample size program (Release 3, Statistical solutions). If $20 \%$ of individuals have a resistant organism, ${ }^{4}$ in a sample of 455 patients with complete outcomes we should be able to detect a difference in symptom resolution of $0.33 \mathrm{SD}$ (1-2 days). There are no clearly established risk factors for prognosis, but we hypothesised that patients' perceptions of communication, ${ }^{2}$ indices of severity ${ }^{7}$ comorbidity, previous operations, ${ }^{1}$ somatic symptoms, ${ }^{6}$ history, and marital status might be important. Based on this and assuming that five to eight variables in a model might explain about $20 \%$ of the variance in outcome, for $80 \%$ power and $\alpha$ of 0.01 (to minimise type I error), we 
Table 1| Mean (SD) duration of symptoms after women with urinary tract infection (UTI) saw doctor or nurse for symptoms rated as moderately bad problem or worse*

\begin{tabular}{|c|c|c|c|c|c|c|c|c|c|c|}
\hline & $\begin{array}{c}\text { Symptom } \\
\text { duration in } \\
\text { days }(n=511)\end{array}$ & $\begin{array}{c}\text { Haematuria } \\
\text { duration } \\
(n=82,16 \%)\end{array}$ & $\begin{array}{c}\text { Dysuria } \\
(n=326,64 \%)\end{array}$ & $\begin{array}{c}\text { Urgency } \\
(n=320,63 \%)\end{array}$ & $\begin{array}{c}\text { Daytime } \\
\text { frequency } \\
(n=397,78 \%)\end{array}$ & $\begin{array}{c}\text { Nocturia } \\
(n=293,57 \%)\end{array}$ & $\begin{array}{c}\text { Offensive } \\
\text { smell } \\
(n=141,28 \%)\end{array}$ & $\begin{array}{c}\text { Abdo pain } \\
(n=238,47 \%)\end{array}$ & $\begin{array}{c}\text { Restricted } \\
\text { activities } \\
(n=214,42 \%)\end{array}$ & $\begin{array}{c}\text { Unwell } \\
(n=241,47 \%)\end{array}$ \\
\hline Overall duration & $3.83(2.97)$ & $1.88(1.75)$ & $2.67(2.26)$ & $3.06(2.54)$ & $3.46(2.59)$ & $3.14(2.50)$ & $2.92(2.46)$ & $3.15(2.57)$ & $2.89(2.59)$ & $3.13(2.62)$ \\
\hline \multicolumn{11}{|l|}{ Antibiotic resistance: } \\
\hline $\begin{array}{l}\text { Sensitive } \\
\text { organism } \\
(n=224)\end{array}$ & $3.32(2.54)$ & $1.78(1.70)$ & $2.24(1.80)$ & $2.48(1.98)$ & $3.03(2.44)$ & $2.52(2.09)$ & $2.13(1.44)$ & $2.61(2.47)$ & $2.68(2.86)$ & $2.71(2.50)$ \\
\hline $\begin{array}{l}\text { Unknown } \\
\text { sensitivity }(n=47)\end{array}$ & $3.32(2.06)$ & $1.64(1.50)$ & $2.39(2.89)$ & $2.63(1.87)$ & $2.78(1.73)$ & $2.21(1.59)$ & $2.59(1.54)$ & $3.08(2.36)$ & $2.09(1.11)$ & $2.83(1.72)$ \\
\hline $\begin{array}{l}\text { Resistant } \\
\text { organism }(n=40)\end{array}$ & $4.73(2.91)$ & $1.0(0.0)$ & $3.52(2.06)$ & $4.04(2.47)$ & $4.15(2.22)$ & $4.04(2.22)$ & $4.38(2.61)$ & $4.78(3.41)$ & $3.88(3.26)$ & $4.18(2.94)$ \\
\hline $\begin{array}{l}\text { UTI, no antibiotic } \\
\text { given }(n=17)\end{array}$ & $4.94(3.82)$ & $3.00(\mathrm{NA})$ & $5.25(3.37)$ & $4.71(4.54)$ & $6.3(3.02)$ & $4.22(3.38)$ & $6.00(1.41)$ & $2.20(1.30)$ & $5.17(3.97)$ & $5.33(4.18)$ \\
\hline $\begin{array}{l}\text { Urethral syndrome } \\
(n=183)\end{array}$ & $4.30(3.42)$ & $2.35(2.11)$ & $3.08(2.79)$ & $3.63(2.99)$ & $3.81(2.81)$ & $3.97(2.91)$ & $4.21(3.51)$ & $3.50(2.45)$ & $2.99(2.10)$ & $3.29(2.70)$ \\
\hline
\end{tabular}

NA=not available (could not be calculated).

*Includes only women with good quality complete diary information for all symptoms and in whom nature of antibiotic resistance could be determined.

needed 312 patients with complete datasets to detect an additional variable that explains a further $3 \%$ of the variance in outcome (and much smaller than $3 \%$ of the variance would probably not be an important variable).

\section{Analysis}

We used Stata version 9 (StataCorp, 2005, College Station, TX). For description and modelling of outcomes we report means rather than medians as with small numbers medians are less sensitive to group differences. We assessed predictors of duration of illness by negative binomial regression (caused by overdispersion of the data) and used linear regression for the data on symptom severity. To assess potential confounding variables, we entered variables significant in univariate analysis $(\mathrm{P}<0.05$, to limit type I error $)$ into multivariate analyses and retained them if they were significant; all the univariate variables were then tested in the model and any further significant variables retained. To assess the pattern of symptoms in the period immediately after the patient had seen the doctor, when symptoms were most severe (days 2-4), we used factor analysis (which identifies related groups of variables), then performed varimax rotation (which creates a solution that maximally separates the factors), and assessed the internal reliability of the scales with Cronbach's $\alpha$ statistic.

\section{RESULTS}

Over $90 \%$ of eligible patients approached agreed to participate: 843 women took part and 839 supplied samples of midstream urine. We had data on antibiotic resistance for 796. We had information on baseline symptoms from 830, some information about duration of symptoms from $684(81 \%)$ women, and 541 (64\%) returned more complete symptom diaries (to allow calculation of severity on a day by day basis). For these 541 women, we had data on antibiotic resistance for 511 (table 1).

\section{Response bias}

The baseline characteristics of those women followed up compared with those who did not provide diary information were similar for key symptoms (urgency, frequency, nocturia, dysuria), which suggests little response bias. Although those who did not return diary information were younger (age $35 v 40$ ), age was not a significant predictor of outcome in the multivariate models and including it in the model made no difference to the estimates.

\section{Sample characteristics}

Of those returning demographic and clinical information, 124/410 (30\%) had a degree or equivalent qualifications, 291/480 (61\%) were married, 129/534 (24\%) were taking oral contraceptives, 54/534 (10\%) were taking hormone replacement therapy, 372/527 (71\%) had had previous episodes of cystitis, and 463/496 (93\%) received an antibiotic. The median number of somatic symptoms reported was three.

Trimethoprim was the most common antibiotic prescribed $(>75 \%)$, the others being cephalosporins, amoxicillin or co-amoxiclav, ciprofloxacin, and nitrofurantoin.

\section{Pattern and duration of symptoms}

Symptoms started on average a median of three days previously (interquartile range 3-7) and 63/478 (13\%) had symptoms for more than 10 days. Previous duration neither predicted duration of symptom after the consultation nor altered the estimates of antibiotic resistance. Table 1 shows the mean duration of more severe symptoms (defined as the duration of days when any symptom in the diary was rated moderately bad or worse is) after the consultation. The most common symptom that patients rated as a moderately bad problem was daytime frequency $(397,78 \%$ ), and more than half the patients also rated their dysuria, urgency, and nocturia as a moderately bad problem; 241 (47\%) patients were considerably unwell, and 214 (42\%) 
Table $2 \mid$ Relation between antibiotic resistance and duration of symptoms rated moderately bad or worse with adjustment for potential confounders. Figures are incidence rate ratios ( $95 \%$ confidence intervals)

\begin{tabular}{|c|c|c|c|c|}
\hline \multirow[b]{2}{*}{ Sensitivity* } & \multicolumn{2}{|c|}{ Univariate analysis $†$} & \multicolumn{2}{|c|}{ Multivariate analysis $\ddagger$} \\
\hline & IRR $(95 \% \mathrm{Cl})$ & $P$ value & IRR $(95 \% \mathrm{Cl})$ & $P$ value \\
\hline Sensitive (mean 3.32 days) & 1.00 & - & 1.00 & - \\
\hline Unknown & $1.00(0.79$ to 1.27$)$ & 0.996 & $1.03(0.81$ to 1.30$)$ & 0.833 \\
\hline Resistant & $1.42(1.12$ to 1.81$)$ & 0.004 & $1.56(1.22$ to 1.99$)$ & $<0.001$ \\
\hline No antibiotic & 1.49 (1.06 to 2.10$)$ & 0.023 & $1.62(1.13$ to 2.31$)$ & 0.008 \\
\hline Urethral syndrome & 1.29 (1.12 to 1.49$)$ & $<0.001$ & $1.33(1.14$ to 1.56$)$ & $<0.001$ \\
\hline \multicolumn{5}{|l|}{ Other predictors: } \\
\hline Positive approach to natural course & 0.93 (0.87 to 0.99$)$ & 0.020 & 0.91 (0.84 to 0.99$)$ & 0.021 \\
\hline Perceived personal relationship & 1.04 (1.00 to 1.07$)$ & 0.043 & 1.05 (1.01 to 1.10$)$ & 0.016 \\
\hline Previous cystitis & $1.26(1.09$ to 1.46$)$ & 0.002 & $1.25(1.07$ to 1.46$)$ & 0.004 \\
\hline Somatic symptom inventory (No of symptoms) & 1.04 (1.03 to 1.06$)$ & $<0.001$ & 1.03 (1.01 to 1.05$)$ & 0.002 \\
\hline Severity of baseline unwell group of symptoms & $1.11(1.07$ to 1.16$)$ & $<0.001$ & 1.07 (1.02 to 1.12$)$ & 0.006 \\
\hline Daytime frequency (No of times) & 1.01 (1.00 to 1.02$)$ & 0.008 & 1.01 (1.00 to 1.02$)$ & 0.005 \\
\hline
\end{tabular}

*Sensitivity groups are compared with patients with sensitive infection who were given antibiotics.

tIf compete data available for univariate analysis are used estimates are: unknown 1.00 (0.81 to 1.25$)$, resistant 1.41 (1.14 to 1.75$)$, no antibiotic 1.32 (0.97 to 1.81 ), urethral syndrome 1.29 (1.13 to 1.47 )

$\ddagger$ All variables that significantly relate to outcome are included in this table and table 3 . Other variables assessed in every model were: age leaving full time education, marital status, number of medical problems, previous duration of symptoms, and perception of doctor communication

(communication and partnership approach, health promotion, interest in effect on life) and health anxiety (Whitely index).

rated restriction of activities a moderately bad problem or worse. Daytime frequency was the longest lasting symptom, but most other symptoms rated as a moderately bad problem lasted three days on average. Around 6\% (38) of women did not rate any symptoms as moderately bad or worse. After consultation, $8 \%$ (55) of women experienced a week or more of symptoms they classed as moderately bad or worse. Among women in whom no urinary tract infection was confirmed (that is, patients with so called "urethral" syndrome) there was a similar pattern of severity of symptoms to those with confirmed infection.

Compared with patients who had an organism sensitive to antibiotics, when we controlled for confounding variables the duration of symptoms rated as a moderately bad problem was 50-60\% longer among patients with antibiotic resistant organisms or who did not receive an antibiotic (tables 1 and 2). Patients with urethral syndrome were less affected. The duration of symptoms rated as moderately bad was also less when the doctor was positive about diagnosis. Symptoms lasted longer in women with frequent somatic symptoms, previous cystitis, and more severe symptoms at baseline. Thus in a woman with higher than median baseline severity $(>3)$, previous cystitis, and higher than the median somatic symptoms $(>3)$, moderately bad or worse symptoms will last twice as long as the average (incidence rate ratio 2.04, 95\% confidence interval 1.61 to 2.57 ) and in a woman with two of these features $64 \%$ longer $(1.64,1.33$ to 2.03$)$. Antibiotic resistance and lack of antibiotic treatment were also associated with greater severity of frequency symptoms (table 3).

\section{DISCUSSION}

In women presenting to primary care with suspected uncomplicated urinary tract infection, the most common symptom rated as a moderately bad problem was urinary frequency and this symptom on average lasted longest; many women also felt considerably unwell and had restricted activities. Patients not given antibiotics and those with antibiotic resistant organisms complained of at least one symptom that was moderately severe or worse lasting five days, and patients rated most individual symptoms as moderately bad or worse for on average four to five days after they saw the doctor or nurse. In patients with sensitive organisms or those with unknown resistance (most of whom would be expected to have sensitive organisms), ${ }^{4}$ duration of symptoms rated as moderately bad and less severe was 50-60\% shorter, and these differences persisted when we adjusted for other confounding variables. Our findings are consistent with previous observations of the association of antibiotic resistance with prolonged more minor symptoms. ${ }^{14}$ The pattern of results, the persistence of the effects when we controlled for confounding, and the size of the effects suggest that both antibiotics and antibiotic resistance are associated with clinically important differences in duration of symptoms.

Patients with the urethral syndrome had symptoms of similar duration and severity to those with confirmed urinary tract infection.

\section{Other predictors of symptom duration}

The finding that a positive approach to diagnosis and prognosis is associated with shorter symptom duration supports previous observational studies and trials. ${ }^{219}$ The finding that a perceived personal relationship is associated with prolonged symptoms is probably due to reverse causality because these patients are more likely to have had previous prolonged and serious illness and altered symptom perception. Patients reporting frequent somatic symptoms are often well known 
Table $3 \mid$ Association of antibiotic resistance and other variables with severity of symptoms in frequency group* at days 2 to 4 (increased day frequency, increased night frequency, and urgency and dysuria)

\begin{tabular}{|c|c|c|c|c|c|c|}
\hline & \multicolumn{3}{|c|}{ Univariate analysis } & \multicolumn{3}{|c|}{ Multivariate analysis } \\
\hline & $\begin{array}{l}\text { Mean (SD) } \\
\text { severity } \dagger\end{array}$ & $\begin{array}{c}\text { Mean difference } \\
(95 \% \mathrm{Cl})\end{array}$ & $P$ value & $\begin{array}{l}\text { Mean (SD) } \\
\text { severity } †\end{array}$ & $\begin{array}{l}\text { Difference } \\
(95 \% \mathrm{Cl})\end{array}$ & $P$ value \\
\hline \multicolumn{7}{|l|}{ Sensitivity } \\
\hline Sensitive $(n=224)$ & $1.52(1.04)$ & - & - & $1.47(0.88)$ & - & - \\
\hline Unknown (n=47) & $1.69(0.98)$ & $0.16(-0.32$ to 0.46$)$ & 0.342 & $1.65(0.88)$ & $0.18(-0.11$ to 0.47$)$ & 0.229 \\
\hline Resistant (n=40) & $2.11(1.16)$ & $0.58(0.22$ to 0.95$)$ & 0.002 & $2.01(0.89)$ & $0.54(0.22$ to 0.87$)$ & $<0.001$ \\
\hline No antibiotic $(n=17)$ & $1.54(1.23)$ & $0.02(-0.51$ to 0.56$)$ & 0.938 & $2.07(0.90)$ & $0.60(0.14$ to 1.05$)$ & 0.011 \\
\hline Urethral syndrome $(n=183)$ & $1.70(1.12)$ & $0.18(-0.03$ to 0.39$)$ & 0.092 & $1.83(0.88)$ & $0.36(0.17$ to 0.56$)$ & $<0.001$ \\
\hline \multicolumn{7}{|l|}{$\beta$ coefficients§ } \\
\hline Positive approach to natural course & - & $-0.04(-0.14$ to 0.06$)$ & 0.417 & - & $-0.18(-0.28$ to -0.08$)$ & 0.001 \\
\hline Perceived personal relationship & 一 & $0.06(0.01$ to 0.11$)$ & 0.030 & - & $0.10(0.05$ to 0.15$)$ & $<0.001$ \\
\hline Somatic symptom inventory & - & 0.07 (0.04 to 0.09$)$ & $<0.001$ & - & $0.03(0.001$ to 0.05$)$ & 0.017 \\
\hline Previous cystitis & - & 0.27 (0.05 to 0.48$)$ & 0.016 & - & 0.19 (0.00 to 0.37$)$ & 0.047 \\
\hline Severity of baseline unwell group of symptoms* & - & $0.22(0.17$ to 0.28$)$ & $<0.001$ & - & $0.08(0.02$ to 0.14$)$ & 0.014 \\
\hline Severity of baseline frequency group of symptoms* & - & $0.39(0.33$ to 0.44$)$ & $<0.001$ & - & $0.40(0.33$ to 0.46$)$ & $<0.001$ \\
\hline \multicolumn{7}{|c|}{$\begin{array}{l}\text { *In factor analysis of severity of symptoms at day } 1 \text { there were two groups of symptoms: "frequency" group (increased day frequency, increased night frequency, and urgency and dysuria) } \\
\text { (Cronbach's } \alpha=0.77 \text { ) and "unwell" group (abdominal pain, restricted activities, and feeling unwell) (Cronbach's } \alpha=0.80 \text { ). At days } 2-4 \text { when symptoms remain most problematic, there was } \\
\text { similar pattern (that is, frequency and unwell groups of symptoms, Cronbach's } \alpha=0.79 \text { and } 0.86 \text {, respectively). Score for frequency symptoms presented as mean of all items. } \\
+0=n o, 6=a s \text { bad as it could be. } \\
\ddagger \text { As in previous tables sensitivity groups are compared with sensitive group given antibiotics as comparison group. If complete data available for univariate analysis are used estimates are: } \\
\text { sensitive } 1.82(1.37) \text {; resistant } 2.37(1.38) \text {; no antibiotic } 1.99(1.40) \text {; urethral syndrome } 1.95(1.37) \text {. } \\
\$ \beta \text { coefficients are estimates of increase in frequency symptoms for one unit increase in independent variable-for example, for every } 1 \text { point increase in rating of positive approach, severity } \\
\text { score decreases by mean of } 0.04 \text {. }\end{array}$} \\
\hline
\end{tabular}

to doctors and are likely to attend more often. ${ }^{6}$ Our results also suggest they are likely to experience or report more prolonged symptoms and that patients with a history of cystitis and more severe baseline symptoms could also be advised that symptoms might take a little longer to settle. Such women - that is, those with numerous somatic symptoms and severe baseline symptoms, particularly if they have a history of cystitis-are arguably a priority group for receipt of antibiotics.

\section{Study limitations and strengths}

Previous studies in acute infection support the validity of self reported diary symptoms. ${ }^{1520}$ The similarity of estimates for antibiotic resistant organisms and no antibiotics also suggests little differential measurement bias. Any non-differential measurement error is likely to provide conservative estimates of group differences. Type I error is unlikely for the main findings as these are highly significant and the pattern is similar for both symptom duration and severity. We had complete results for 500 patients and so had reasonable power for most comparisons. The numbers who received no treatment were low and so negative comparisons for this group must be treated with caution.

We controlled for a range of confounders associated with the patients and doctors that have not been assessed in previous studies and found some confounding (a 10-30\% change in estimates). By comparing the impact of management with an antibiotic to which the infection is resistant and no offer of antibiotics - which we would expect to be similar - we have also helped to clarify what outcomes are likely to be affected by antibiotic treatment and antibiotic resistance.
Most of the women invited to join the study took part (under 10\% declined to participate). Loss of diary information during follow-up was not related to key baseline variables, and the duration of more severe symptoms among those who provided brief information about symptoms (3.4 days) was similar to the duration among those who provided complete diaries, so significant response bias among those returning complete diaries is less likely. Furthermore we controlled for a wide range of confounding variables so it seems unlikely that the characteristics of women for whom we could not get complete sets of data is a limiting factor.

\section{Generalisability}

The mixed locations of the practices in rural and urban settings and the range of demographics among women should make these results generalisable within the UK.

\section{Conclusion}

At presentation most women with urinary tract infection have multiple symptoms that they rate as a moderately bad problem or worse, and half feel unwell and have considerable restriction in daily activities. Antibiotic resistance and not providing antibiotics are associated with $50-60 \%$ longer duration of more severe symptoms and more severe symptoms of frequency. Patients with a history of cystitis, frequent somatic symptoms, and severe baseline symptoms can be given a realistic indication that more severe symptoms might last longer than the average three days. Doctors should also probably remain positive about the natural course for patients with suspected urinary tract infection. 


\section{WHAT IS ALREADY KNOWN ON THIS TOPIC}

In patients with urinary tract infection antibiotic resistance is associated with delayed resolution of symptoms, but the impact on severe symptoms is unknown

\section{WHAT THIS STUDY ADDS}

Antibiotic resistance, or not prescribing antibiotics, is associated with an increase of over $50 \%$ in more severe symptoms

Patients with a history of cystitis, frequent somatic symptoms (high somatisation), and severe symptoms at baseline are likely to have severe symptoms lasting longer than three days

Faster symptom resolution is associated with doctors and nurses being positive about the diagnosis and natural course of the infection
5 Richards D, Toop L, Chambers S, Fletcher L. Response to antibiotics of women with symptoms of urinary tract infection but negative dipstick urine test results: double blind randomised controlled trial. BMJ 2005;331:143.

6 Little P, Somerville J, Williamson I, Warner G, Moore M, Wiles R, et al. Psycho-social, lifestyle and health status variables in predicting high attendance among adults. Br J Gen Pract 2001;51:987-94.

7 Little P, Gould C, Williamson I, Warner G, Gantley M, Kinmonth A. Clinical and psychosocial predictors of illness duration from a randomised controlled trial of prescribing strategies for sore throat. BMJ 1999;319:736-7.

8 Nickel J, Lee J, Grantmyre J, Polygensis D. Natural history of urinary tract infection in a primary care environment in Canada. Can J Urol 2005;2728-37.

9 Standing Medical Advisory Committee (SMAC), Sub-Group on Antimicrobial resistance. The path of least resistance. Department of Health, 1998.

10 Little P, Turner S, Rumsby K, Warner G, Moore M, Lowes JA, et al. Urinary tract infection: development and validation, randomised trial, economic analysis, observational cohort and qualitative study. Health Technol Assess 2009;13:19.

We are very grateful for the time given by patients, general practitioners, and nurses. We are grateful to Bayer for provision of the 8SG strips. Contributors: PL and MVM had the original idea for the study. All authors developed the protocol. ST ran the study on a day to day basis. KR managed the database. PL and MM performed the analysis. PL and MM are guarantors. All authors contributed to writing the paper Funding: This study was funded by the health technology programme of UK NHS Research and Development.

Competing interests: JAL has been paid to attend consultancy workshops by Bayer and is currently working in collaboration with Bayer in an unpaid capacity.

Ethical approval: This study was approved by the South West MReC ethics committee and informed consent was given by all patients. Data sharing: The authors are happy to share data when appropriate-for example, for meta-analyses; requests to the corresponding author.

1 Butler C, Hillier S, Roberts Z, Dunstan F, Howard A, Palmer S. Antibiotic resistant infections in primary care are symptomatic for longer and increase workload. Br J Gen Pract 2006:56:686-92.

2 Little P, Everitt H, Williamson I, Moore M, Warner G, Gould C, et al. An observational study of patient-centredness in primary care, and its relationship to outcome. BMJ 2001;323:908-11.

3 Christiaens T, DeMeyere M, Verschraegen G, Peersman W, Heytens S, De Maeseneer J. Randomised controlled trial of nitrofurantoin versus placebo in the treatment of uncomplicated urinary tract infection in adult women. Br/Gen Pract 2002;52:729-34.

4 McNulty C, Richards J, Livermore D, Little P, Charlett A, Freeman E, et al. Clinical relevance of laboratory reported antibiotic resistance in acute uncomplicated urinary tract infection in primary care. $J$ Antimicrob Chemother 2006;58:1000-8.
11 Little P, Moore M, Turner S, Rumsby K, Warner G, Lowes A, et al. Effectiveness of five different approaches in the management of urinary tract infection: a randomised controlled trial. $B M$ J 2010; doi:10.1136/bmj.c199.

12 Hummers-Pradier E, Kochen M. Urinary tract infections in adult general practice patients. BrJ Gen Pract 2002;52:752-9.

13 Childs S, Egan R. Bacteriuria and urinary infections in the elderly. Urologic Clinics of North America 1996;23(1):43-54.

14 Dobbs FF, Fleming DM. A simple scoring system for evaluating symptoms, history and urine dipstick testing in the diagnosis of urinary tract infection. J R Coll Gen Pract 1987;37:100-4.

15 Watson L, Little P, Williamson I, Moore M, Warner G. Validation study of a diary for use in acute lower respiratory tract infection. Fam Pract 2001;18:553-4.

16 Howie J, Heaney D, Maxwell M, Walker J, Freeman G, Rai H. Quality at general practice consultations: cross sectional survey. $B M$ 1999;319:738-43.

17 European Confederation of Laboratory Medicine. ECLM-European Urinanalysis Group European Analysis guidelines. Scand J Clin Lab Invest 2000;60:1-96.

18 Little P, Turner S, Rumsby K, Warner G, Moore M, Lowes A, et al. Developing clinical rules to predict urinary tract infection in primary care settings: sensitivity and specificity of near patient tests (dipsticks) and clinical scores. Br J Gen Pract 2006;529:606-12.

19 Thomas KB. General practice consultations: is there any point being positive? BMJ 1987;294:1200-2.

20 Little PS, Williamson I, Warner G, Gould C, Gantley M, Kinmonth AL. An open randomised trial of prescribing strategies for sore throat. BMJ 1997;314:722-7.

Accepted: 15 November 2009 